Article

\title{
Copper-Catalyzed Synthesis of Unsymmetrical Diorganyl Chalcogenides (Te/Se/S) from Boronic Acids under Solvent-Free Conditions $\ddagger$
}

\author{
Sumbal Saba ${ }^{1,2,+}$ (D), Giancarlo Vaccari Botteselle ${ }^{3}$, Marcelo Godoi ${ }^{4}$, Tiago Elias Allievi Frizon 5 , \\ Fábio Zazyki Galetto ${ }^{1}$, Jamal Rafique ${ }^{1, *,+}$ (1) and Antonio L. Braga ${ }^{1, *}$ \\ 1 Departamento de Química, Universidade Federal de Santa Catarina (UFSC), Florianopolis SC 88040-900, \\ Brazil; sumbal6s@gmail.com (S.S.); galetto.f.z@ufsc.br (F.Z.G.) \\ 2 Department of Chemistry, Shaheed Benazir Bhutto Women University, Peshawar 25000, Pakistan \\ 3 Laboratório de Pesquisa Química, CECE, Universidade Estadual do Oeste do Paraná UNIOESTE, \\ Toledo PR 85819-110, Brazil; gian.botteselle@gmail.com \\ 4 Escola de Química e Alimentos, Campus Santo Antônio da Patrulha, Universidade Federal do Rio Grande, \\ Santo Antônio da Patrulha RS 96201-900, Brazil; marcelogodoi@furg.br \\ 5 Campus Araranguá, Universidade Federal de Santa Catarina (UFSC), Araranguá SC 88905-120, Brazil; \\ tiagofrizon@gmail.com \\ * Correspondence: jamal.chm@gmail.com (J.R.); braga.antonio@ufsc.br (A.L.B.); Tel.: +55-48-3721-3651 (J.R.); \\ $+55-48-3721-6427$ (A.L.B.) \\ + These authors contributed equally to this work. \\ $\ddagger$ Dedication: A.L.B., S.S. and J.R. dedicate this paper with gratitude to Joao Valdir Comasseto for his notable \\ contributions in organochalcogen chemistry over the past 40 years.
}

Received: 4 July 2017; Accepted: 16 August 2017; Published: 18 August 2017

\begin{abstract}
The efficient and mild copper-catalyzed synthesis of unsymmetrical diorganyl chalcogenides under ligand- and solvent-free conditions is described. The cross-coupling reaction was performed using aryl boric acids and 0.5 equiv. of diorganyl dichalcogenides (Te/Se/S) in the presence of $3 \mathrm{~mol} \%$ of $\mathrm{CuI}$ and 3 equiv. of DMSO, under microwave irradiation. This new protocol allowed the preparation of several unsymmetrical diorganyl chalcogenides in good to excellent yields.
\end{abstract}

Keywords: selenide; telluride; boronic acid; solvent-free; cross-coupling; CuI; selenium; tellurium

\section{Introduction}

Transition metal-catalyzed coupling reactions are among the most commonly applied protocols for the preparation of various target molecules under mild conditions [1,2]. In this regard, the construction of new carbon-carbon and carbon-heteroatom bonds have been described, notably through catalytic processes employing a metal as the catalyst [3-7].

In addition, catalytic transformations have been widely applied in organochalcogen chemistry for the preparation of diorganyl-tellurides, -selenides and -sulfides, which are interesting target molecules and valuable synthetic intermediates for several transformations in modern organic synthesis [8-10]. Besides the synthetic applications, these types of molecules have shown relevant biological properties, such as antitumor, antioxidant, antiviral, antimicrobial and neuroprotective effects [11-19]. Organochalcogen compounds also have noteworthy applications in materials science [20-22].

Due to their considerable importance, the development of new and greener methods for the preparation of organochalcogenides $(\mathrm{S}, \mathrm{Se}$ and $\mathrm{Te}$ ) has been widely investigated by several research groups, and a number of methodologies have been reported [23-30]. The most common procedures described for the preparation of alkyl/aryl chalcogenides involve: (a) the reaction between alkyl/aryl 
dichalcogenide precursors and organometallic reagents [31-34]; (b) the transition metal-catalyzed reaction of halides with chalcogenols or diorganyl dichalcogenides in the presence of a base or reducing agents [35-37]; and (c) synthesis through the $\mathrm{C}-\mathrm{H}$ activation of arenes [38-41].

On the other hand, organoboronic acids have been used as suitable reagents for various cross-coupling reactions since they are stable, readily available and compatible with different functional groups. They can also be employed in the boron-to-metal exchange reaction using a catalytic amount of metal [42-44]. Thus, organoboronic acids have become interesting and appropriate alternative compounds for the preparation of symmetrical and unsymmetrical diaryl organochalcogenides via cross-coupling transformations.

In this regard, Wang and Tanigushi, independently reported the preparation of diorganyl chalcogenides from organoboronic acids and diorganyl dichalcogenides using copper as a catalyst, in the presence of a ligand and solvent $[45,46]$. Since their reports, modern studies have been described to improve this kind of transformation. In particular, metals have been efficiently applied as catalysts for the cross-coupling of diorganyl chalcogenides with boronic acids, for instance, employing indium [47], iron [48], copper [49-55] and silver [56].

However, most of these procedures have their particular drawbacks, such as the use of ligands, reducing agents, air sensitivity, long reaction time, and/or the use of toxic solvents. In addition, some of these methods are not applicable for the synthesis of unsymmetrical organotellurides, which are also very important from the synthetic point of view.

Alternatively, we have recently reported suitable methods for the synthesis of unsymmetrical chalcogenides, using the iodine/DMSO system under microwave irradiation to generate, in situ, electrophilic species of chalcogenyl iodide (RYI) [57]. Similarly, Park and coworkers reported an ultrasound-assisted synthesis of diaryl ditellurides [58]. Nonetheless, the development of new methodologies employing boronic acids and dichalcogenides as starting materials, associated with metal catalysis instead of use RYI species, is still highly desirable. Furthermore, the use of diorganyl dichalcogenides avoids the use of toxic RXH compounds $(X=T e, S e, S)$.

Thus, in connection with our continuing interest in designing and developing eco-friendly processes and cross-coupling reactions, [59-64], herein we describe a straightforward method for the synthesis of unsymmetrical diorganyl-chalcogenides (S, Se and Te) through the reaction of boronic acids and half equiv. of diorganyl dichalcogenides, employing $\mathrm{CuI}$ as a catalyst, under solvent- and ligand-free conditions (Scheme 1).

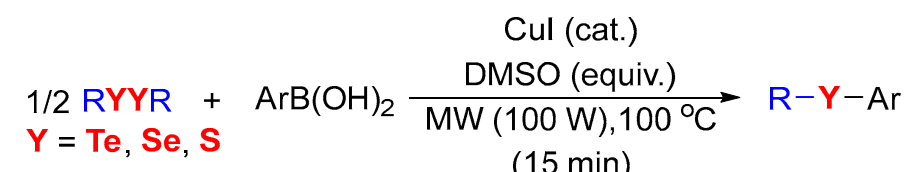

(15 $\mathrm{min})$

Scheme 1. Solvent- and ligand-free synthesis of unsymmetrical diorganyl chalcogenides catalyzed by CuI.

\section{Results and Discussion}

In order to optimize the reaction conditions, diphenyl ditelluride (1a) and 4-methoxyphenyl boronic acid (2a) were used as model substrates (Table 1). Firstly, we evaluated the catalyst loading in the reaction system (entries 1-4). When the reaction was carried out with $1.0 \mathrm{~mol} \%$ of catalyst the desired product was obtained with only $43 \%$ yield (entry 1 ). However, on increasing the catalyst to $2.0 \mathrm{~mol} \%$, the yield of the reaction increased to $71 \%$ (entry 2). Notably, when the amount of CuI was increased to $3.0 \mathrm{~mol} \%$ the corresponding product 3 a was achieved with $90 \%$ yield (entry 3 ). No change in the yield was observed when the catalyst loading was increased to $4.0 \mathrm{~mol} \%$ (entry 4 ).

Next, we investigated the influence of the copper source on the reaction system (entries 5-10). In general, copper halides showed better catalytic activity. For instance, when we employed $\mathrm{CuCl}_{2}$ and $\mathrm{CuO}$ under the same reaction conditions, the desired organotelluride was synthesized in yields of 
72 and $61 \%$, respectively (entries 7 vs. 9). Furthermore, $\mathrm{CuO}$ nanoparticles were less effective than copper iodide, affording the desired product in lower yield (entry 10). Considering our previous work [57], when 3\% molecular iodine was used as catalyst, 3a was obtained in 69\% yield (entry 11). The reaction in the absence of catalyst provided 3a in only trace amounts, which emphasizes the notable activity of $\mathrm{CuI}$ in this kind of transformation (entry 12).

Table 1. Optimization of reaction conditions ${ }^{\text {a }}$.

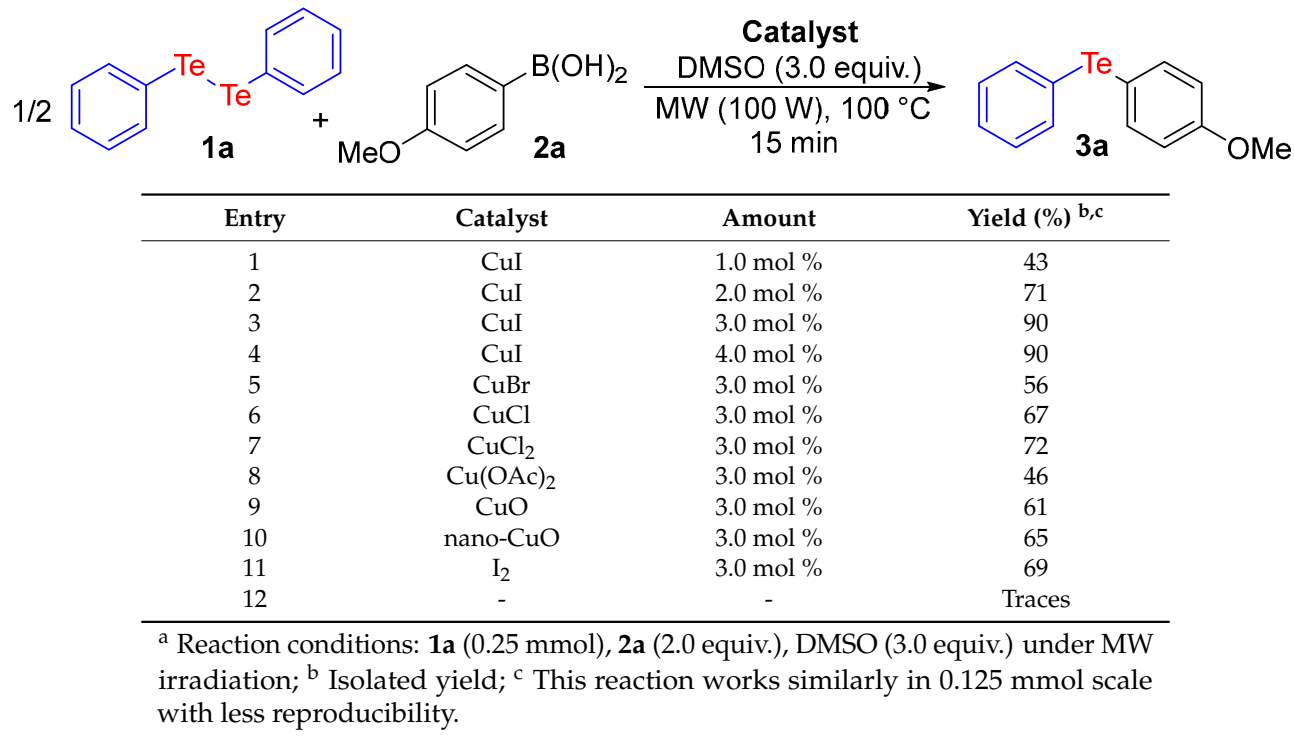

With the best catalyst in hand, we investigated the influence of other reaction parameters (temperature, time and power) as well as the effect of the additive/oxidant (Table 2).

Table 2. Optimization of reaction conditions ${ }^{\mathrm{a}}$.

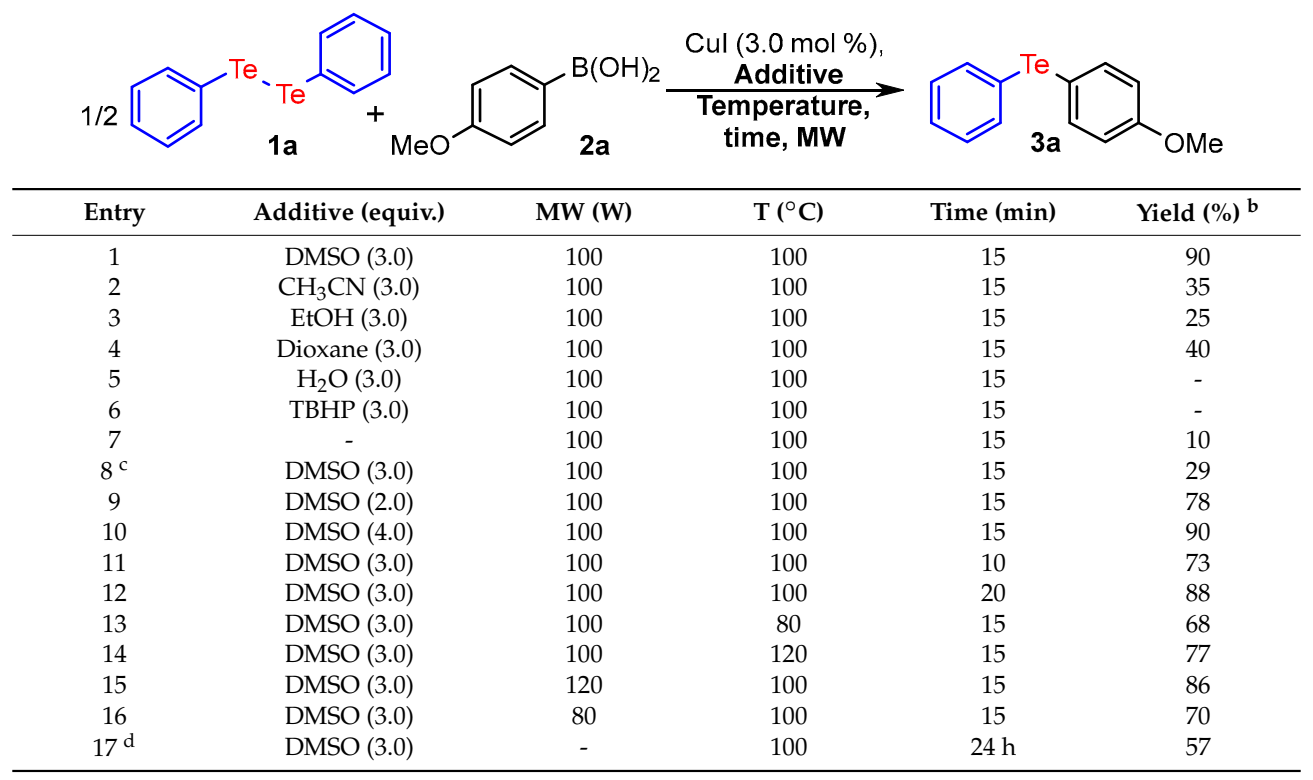

a Reaction conditions: 1a ( $0.25 \mathrm{mmol}), 2 \mathrm{a}$ (2.0 equiv.), CuI (3.0 mol \%), DMSO (3.0 equiv.) under MW irradiation; ${ }^{\mathrm{b}}$ Isolated yield; ${ }^{\mathrm{c}}$ Reaction under argon atmosphere; ${ }^{\mathrm{d}}$ Conventional heating.

In this regard, we initially evaluated the influence of a series of additives $\left(\mathrm{CH}_{3} \mathrm{CN}, 1,4\right.$-dioxane, $\mathrm{EtOH}, \mathrm{H}_{2} \mathrm{O}$ and TBHP). Of these, DMSO was established as the best option, providing the desired product in very high yield in comparison with other additives (entry 1 vs. 2-6). Furthermore, a considerable decrease in the yield was observed when the reaction was carried out either in the 
absence of an additive or under inert atmosphere, highlighting the need for an oxidant medium for this cross-coupling transformation (entries 7 and 8). In relation to the loading of DMSO, it was observed that 3.0 equiv. was the best alternative, since no improvement in the yield was observed when the amount of this additive was modified (entries 9 and 10).

The influence of the reaction time and temperature was also evaluated (entries 11-14). The screening of these parameters revealed that $15 \mathrm{~min}$ and $100{ }^{\circ} \mathrm{C}$ were the most appropriate choice for this protocol, as seen in entry 1.

Finally, the effect of the radiation power of the microwave was investigated and increasing the power to $120 \mathrm{~W}$ did not change the reaction yield significantly (entry 15). However, when the level of power was decreased to $80 \mathrm{~W}$ the desired product 3a was delivered in lower yield (entry 16). In addition, we carried out the reaction under conventional heating and, even after a long reaction time, a decrease in the yield of the product 3a was observed (entry 17). This result indicates that microwave irradiation offers advantages over conventional heating, for this transformation.

Having determined the best reaction conditions, we explored the scope of our protocol by employing various diorganyl ditellurides 1 and arylboronic acids $\mathbf{2}$ (Scheme 2). Firstly, we evaluated the effect of different groups attached to the aryl ring of boronic acid (Scheme 2).

The electronic characteristics of the substituent of boronic acid attached to the aromatic ring (electron donor or acceptor) and its steric hindrance did not appear to affect the performance of the transformation, and the corresponding products $3 a-\mathbf{e}$ were obtained in very good yields. Also, 2-naphthylboronic acid reacted very well with diphenyl ditelluride (1a) under the optimized conditions, affording the corresponding product $3 \mathrm{f}$ in very good yield.

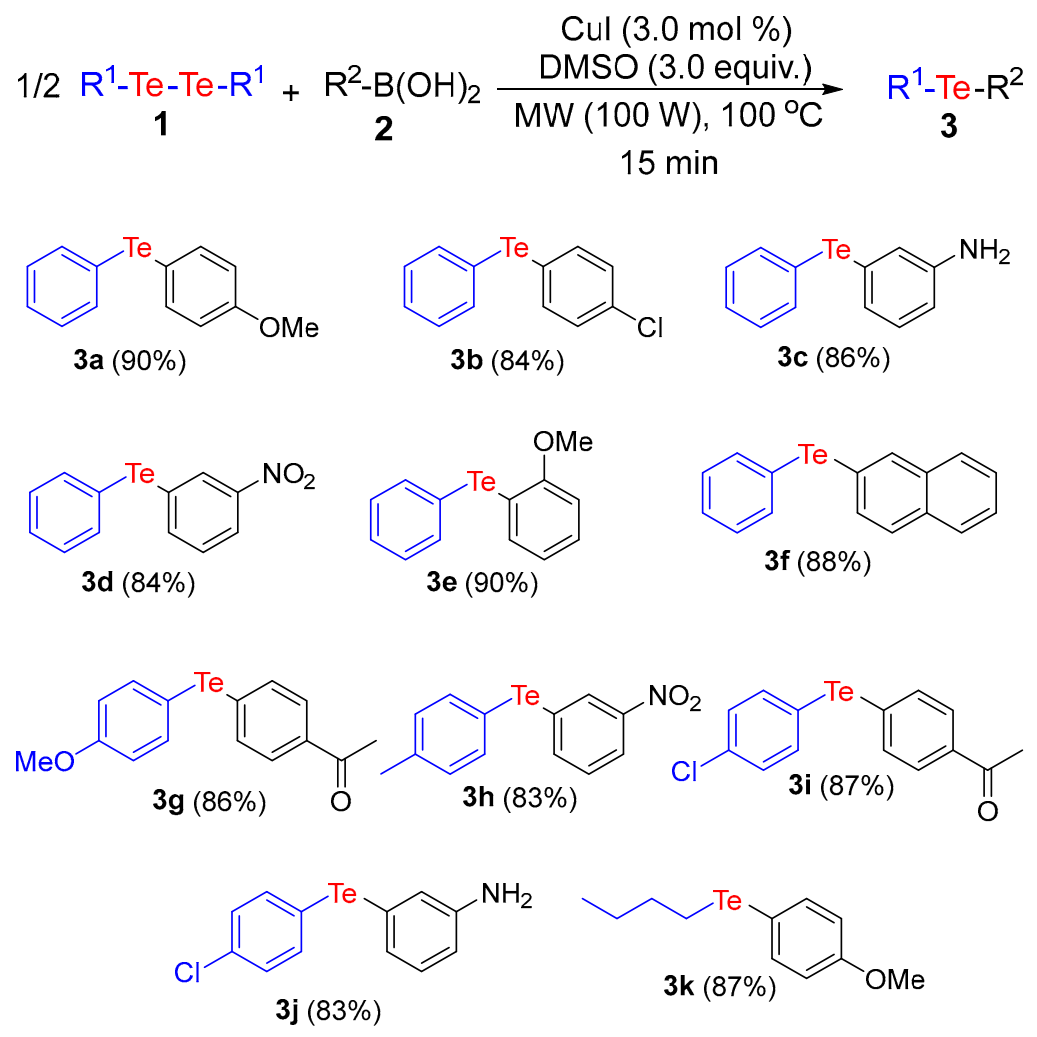

Scheme 2. Synthesis of unsymmetrical organotellurides a,b ${ }^{a}$ Reaction conditions: $\mathbf{1}(0.25 \mathrm{mmol})$, $2(0.5 \mathrm{mmol})$ in the presence of $\mathrm{CuI}(3.0 \mathrm{~mol} \%)$ and DMSO (3.0 equiv.) applied for $15 \mathrm{~min}$ at $100{ }^{\circ} \mathrm{C}$ with $100 \mathrm{~W}$ of MW-irradiation; ${ }^{\mathrm{b}}$ Isolated yields.

We further evaluated the generality of the reaction regarding the ditellurides $\mathbf{1}$, employing electron-withdrawing or electron-donating groups. In general, the reaction proceeded very well in 
both cases, furnishing the corresponding unsymmetrical tellurides $3 \mathbf{g}-\mathbf{j}$ in $83-87 \%$ yields. For instance, the reaction of para-chlorobenzene ditelluride with meta-substituted aryl boronic acid delivered the respective compound $\mathbf{3} \mathbf{j}$ in $83 \%$ yield. Moreover, an aliphatic ditelluride, in this case dibutyl ditelluride, was also a suitable substrate for this cross-coupling process, furnishing the product $3 \mathbf{k}$ in $87 \%$ yield.

The successful preparation of unsymmetrical diorganyl tellurides 3 by a copper-catalyzed transformation prompted us to expand this approach to the synthesis of unsymmetrical diorganyl selenides (Scheme 3). To evaluate the electronic effects, we first verified the influence of the substituent attached at the para position of the aromatic ring of the boronic acid. The treatment of diphenyl diselenide with different para-substituted aryl boronic acids provided the respective products $\mathbf{6 a}$ and $\mathbf{6 b}$ in $87 \%$ and $85 \%$ yields, respectively.

Similarly, the reaction was not sensitive to steric effects, since the treatment of diphenyl diselenide with aryl boronic acid containing a methyl group at the ortho position of the aromatic ring provided the respective product $6 \mathrm{c}$ in $89 \%$ yield. Subsequently, a series of different diselenides, including aliphatic and aromatics diselenides, were converted into the corresponding products in very good yields. For example, when we reacted $p$-chlorobenzene diselenide with 4-methoxyphenylboronic acid the product $6 \mathrm{e}$ was obtained in $89 \%$ yield.

We also evaluated the applicability of our protocol to the preparation of unsymmetrical sulfides, under the same reaction conditions. Remarkably, diphenyl disulfide and $m$-chlorophenyl disulfide reacted smoothly with $p$-methoxyphenyl boronic acid, leading to the respective products $7 \mathbf{a}$ and $\mathbf{7 b}$ in $71 \%$ and $68 \%$ yields, respectively. This slight decrease in the yield values could be associated with the lower reactivity of diaryl disulfides when compared to ditellurides or diselenides analogues, which are much more easily cleaved than disulfides [65].

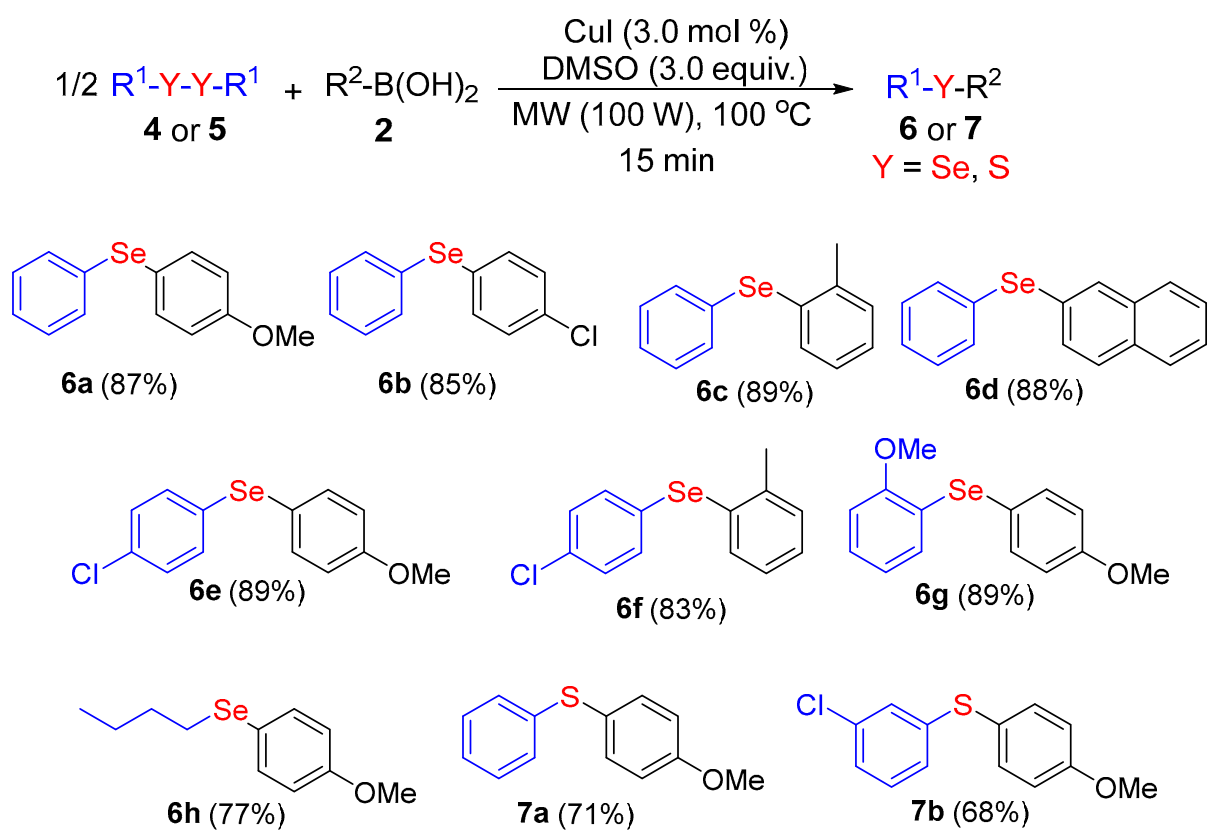

Scheme 3. Synthesis of unsymetrical organoselenides and sulfides ${ }^{\mathrm{a}, \mathrm{b}}$. ${ }^{\mathrm{a}}$ Reaction conditions: 4 or $5(0.25 \mathrm{mmol}), 2(0.5 \mathrm{mmol})$ in the presence of $\mathrm{CuI}(3.0 \mathrm{~mol} \%)$ and DMSO (3.0 equiv.) for $15 \mathrm{~min}$ at $100{ }^{\circ} \mathrm{C}$ and $100 \mathrm{~W}$ of MW-irradiations; ${ }^{\mathrm{b}}$ Isolated yields.

In addition, in order to investigate the synthetic utility of this methodology, we evaluated whether the reaction could be performed with boronic acid at the gram scale under the optimized conditions (Scheme 4). To our delight, the corresponding product 3a was obtained in $85 \%$ yield, which is very significant from a synthesis point of view, since this methodology can thus be used to prepare unsymmetrical diorganyl chalcogenides on a larger scale. 


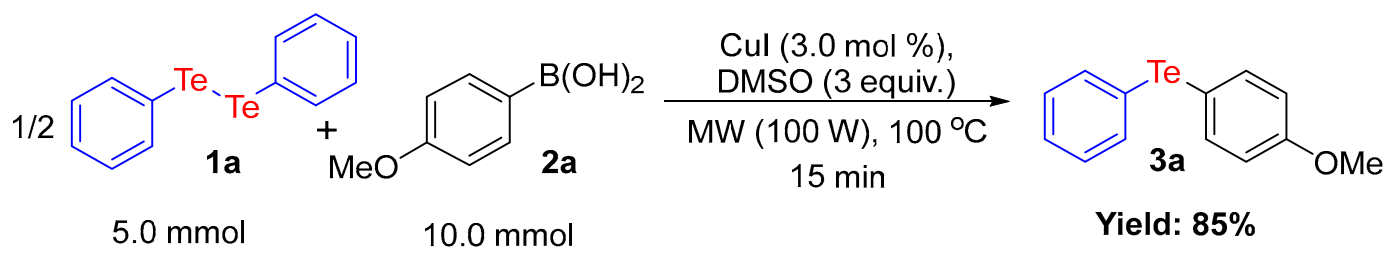

Scheme 4. Scale-Up of the reaction.

In order to gain further insight into the reaction mechanism, control experiments were performed (Scheme 5). Firstly, to evaluate the possibility of a radical path, we performed the standard reaction in the presence of 3.0 equiv. of 2,2,6,6-tetramethyl-1-piperidinyloxy (TEMPO) as a radical inhibitor (Scheme 5a). The presence of TEMPO did not affect the reaction yield, which suggests that radical intermediates are not involved in this protocol. Next, we verified the influence of oxidant species on this transformation. When the reaction was carried out under argon atmosphere, an abrupt decrease was noted and the telluride $3 a$ was delivered in only $29 \%$ yield (Scheme 5 b). In contrast, when diphenyl ditelluride was treated with phenyl boronic under $\mathrm{O}_{2}$ atmosphere, the corresponding product was obtained in $93 \%$ yield (Scheme $5 \mathrm{c}$ ). These results suggest that oxygen is mainly responsible for the oxidation steps and DMSO is most likely an important additive in terms of improving the reaction yield.

$1 / 2$<smiles>COc1ccc([18O]c2ccccc2[Te]c2ccccc2)cc1</smiles>

$1 / 2$<smiles>COc1ccc([18O]c2ccccc2[Te]c2ccccc2)cc1</smiles>

$1 / 2$<smiles>COc1ccc([18O]c2ccccc2[Te]c2ccccc2)cc1</smiles>

TEMPO

Cul $(3.0 \mathrm{~mol} \%)$,

$15 \mathrm{~min}$<smiles>COc1ccc([Te]c2ccccc2)cc1</smiles>

3a $89 \%$

\section{Inert atmosphere}

Cul $(3.0 \mathrm{~mol} \%)$

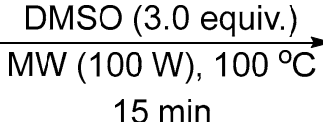<smiles>COc1ccc([Te]c2ccccc2)cc1</smiles>

3a $29 \%$

\section{$\mathrm{O}_{2}$ atmosphere}

Cul $(3.0 \mathrm{~mol} \%)$,

\section{$\underset{\mathrm{DMSO}(3.0 \text { equiv. })}{\mathrm{MW}(100 \mathrm{~W}), 100^{\circ} \mathrm{C}}$}

$15 \mathrm{~min}$

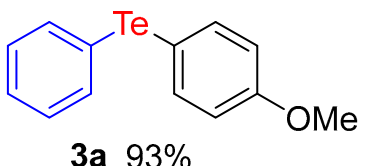

(c)

Scheme 5. Control experiments for reaction mechanism. (a) Reaction in the presence of radical inhibitor; (b) Reaction under inert atmosphere; (c) Reaction under oxygen atmosphere.

On the basis of these results and in accordance with previous reports [66,67], a plausible reaction pathway is illustrated in Scheme 6. In this pathway the catalyst cycle begins with the generation of species A through the reaction between $\mathrm{CuI}$ and boronic acid [66]. Subsequently, this specie reacts with diorganyl dichalcogenide to afford the desired product and the intermediate $\mathbf{B}$. The specie $\mathbf{B}$ is then oxidized to give the $\mathrm{Cu}(\mathrm{II})$ intermediate $\mathbf{C}[66,67]$. In the next step, another equiv. of boronic acid would reacts with this intermediate to furnish the intermediate $\mathbf{D}$. Lastly, a second equiv. of the desired product is obtained through an elimination reaction step and, consequently, the catalyst is regenerated. 


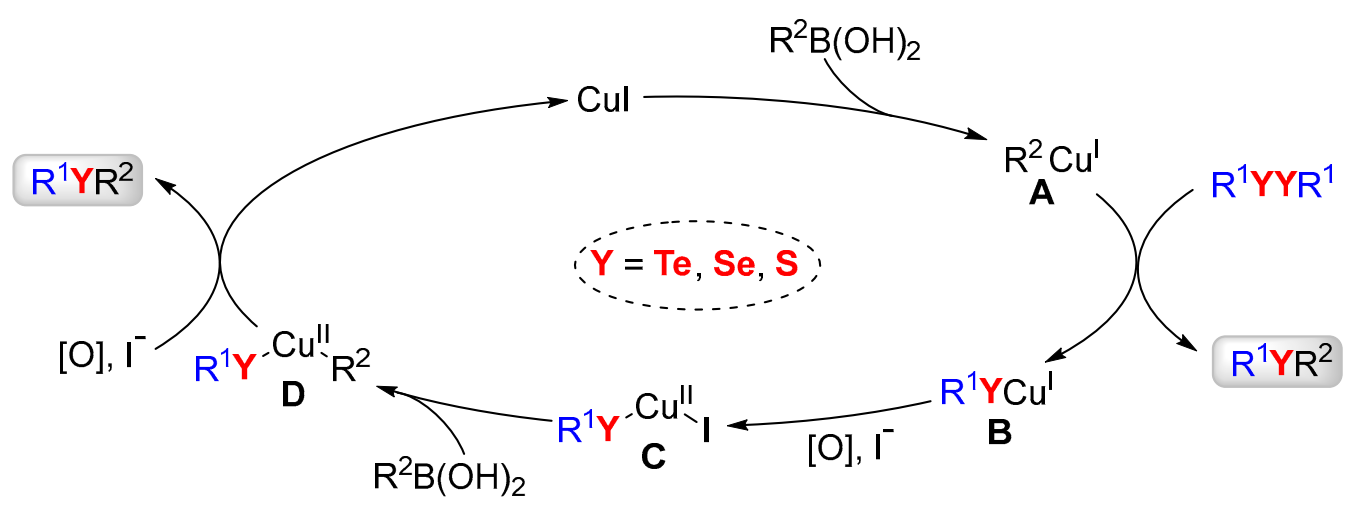

Scheme 6. A plausible reaction pathway.

\section{Materials and Methods}

\subsection{General Information}

${ }^{1} \mathrm{H}$ - and ${ }^{13} \mathrm{C}-\mathrm{NMR}$ spectra were obtained at 200/50 MHz on an AC-200 NMR spectrometer (Bruker, Rheinstetten, Germany) or at $400 / 100 \mathrm{MHz}$ on an AS-400 NMR spectrometer (Varian, Palo Alto, CA, USA). Spectra were recorded in $\mathrm{CDCl}_{3}$ solutions. Chemical shifts are reported in ppm, referenced to the solvent peak of $\mathrm{CDCl}_{3}$ or tetramethylsilane (TMS) as the external reference. Data are reported as follows: Chemical shift $(\delta)$, multiplicity, coupling constant $(J)$ in hertz and integrated intensity. Abbreviations to denote the multiplicity of a particular signal are: s (singlet), $\mathrm{d}$ (doublet), t (triplet), q (quartet), quint (quintet), sext (sextet) and m (multiplet). The NMR spectra are found in the Supplementary Materials. The melting points were determined using a model MQRPF-301 digital apparatus (Microquimica, Palhoça, Brazil) equipped with a heating plate. Column chromatography was performed using silica gel (230-400 mesh). Thin layer chromatography (TLC) was performed using silica gel $\mathrm{GF}_{254}$ plates with $0.25 \mathrm{~mm}$ thickness (Merck, Darmstadt, Germany). For visualization, TLC plates were either placed under ultraviolet light or stained with iodine vapor and acidic vanillin. Most reactions were monitored by TLC for the disappearance of the starting material.

Unless otherwise stated, all reactions were carried out in an open atmosphere. All reagents and solvents were obtained from commercial sources and used without further purification. All reactions were performed in $10 \mathrm{~mL}$ sealed glass tubes in a commercially available monomode microwave reactor (CEM, Matthews, NC, USA) with IR monitoring and a non-invasive pressure transducer. Reagents and solvents were handled using standard syringe techniques. The yields are based on isolated compounds after purification.

\subsection{General Procedure for the Synthesis of Unsymmetrical Organochalcogenides under MW Irradiation}

A mixture of aryl boronic acid $(0.5 \mathrm{mmol})$, dichalcogenide $(0.25 \mathrm{mmol}), \mathrm{CuI}(3 \mathrm{~mol} \%, 1.5 \mathrm{mg})$, and DMSO (3 equiv., $59 \mathrm{mg}$ ) was placed in a glass tube. The tube was sealed with a pressure lock and the mixture was heated in air at $100{ }^{\circ} \mathrm{C}$ for $15 \mathrm{~min}$ with the aid of an initial MW irradiation of $100 \mathrm{~W}$ in a CEM Discover MW reactor. When the reaction was finished, the crude mixture was cooled to room temperature, diluted with ethyl acetate $(10 \mathrm{~mL})$, and washed with water $(3 \times 5 \mathrm{~mL})$. The organic phase was separated, dried over $\mathrm{MgSO}_{4}$, and concentrated under vacuum. The residue was purified by flash chromatography on silica gel using hexane or a mixture of ethyl hexane/acetate (99:1) as the eluent.

Analytical Data of Products $3 \mathbf{a}-\mathbf{k}, \mathbf{6 a}-\mathbf{h}$ and $7 \mathbf{a}, \mathbf{b}$

(4-Methoxyphenyl)(phenyl)tellane (3a). Yield: $0.140 \mathrm{~g}(90 \%)$; white solid; m.p.: 59-61 ${ }^{\circ} \mathrm{C}$ (lit.: [48] 60-62 $\left.{ }^{\circ} \mathrm{C}\right) ;{ }^{1} \mathrm{H}-\mathrm{NMR}\left(200 \mathrm{MHz}, \mathrm{CDCl}_{3}, \mathrm{ppm}\right) \delta=7.73(\mathrm{~d}, \mathrm{~J}=8.8 \mathrm{~Hz}, 2 \mathrm{H}, \mathrm{H}-\mathrm{Ar}), 7.62-7.42$ (m, 2H, H-Ar),

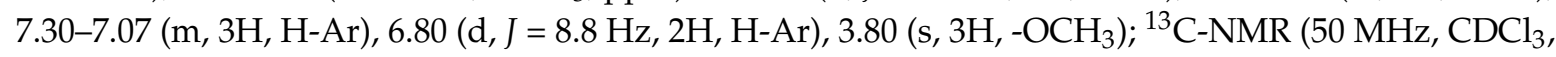
ppm) $\delta=160.1,141.3,136.5,129.4,127.4,116.0,115.6,103.3,55.3$. 
(4-Chlorophenyl)(phenyl)tellane (3b). Yield: $0.133 \mathrm{~g}(84 \%)$; yellow oil; [ 68$]^{1} \mathrm{H}-\mathrm{NMR}\left(200 \mathrm{MHz}, \mathrm{CDCl}_{3}\right.$, ppm) $\delta=7.63-7.57$ (m, 2H, H-Ar), 7.52-7.46 (m, 2H, H-Ar), 7.21-7.05 (m, 5H, H-Ar); ${ }^{13} \mathrm{C}-\mathrm{NMR}(50 \mathrm{MHz}$, $\left.\mathrm{CDCl}_{3}, \mathrm{ppm}\right) \delta=139.3,138.2,134.4,129.8,129.7,128.2,114.5,112.5$.

3-(Phenyltellanyl)aniline (3c). Yield: $0.128 \mathrm{~g}$ (86\%); yellow oil; [57] ${ }^{1} \mathrm{H}-\mathrm{NMR}\left(200 \mathrm{MHz}, \mathrm{CDCl}_{3}, \mathrm{ppm}\right)$ $\delta=7.57(\mathrm{~d}, J=8.4 \mathrm{~Hz}, 2 \mathrm{H}, \mathrm{H}-\mathrm{Ar}), 7.56-6.99(\mathrm{~m}, 6 \mathrm{H}, \mathrm{H}-\mathrm{Ar}), 6.60-6.55(\mathrm{~m}, 1 \mathrm{H}, \mathrm{H}-\mathrm{Ar}), 3.62\left(\mathrm{~s}, 2 \mathrm{H},-\mathrm{NH}_{2}\right)$; ${ }^{13} \mathrm{C}-\mathrm{NMR}\left(50 \mathrm{MHz}, \mathrm{CDCl}_{3}\right.$, ppm) $\delta=147.3,138.0,130.3,129.6,128.2,127.8,124.4,115.4,114.9,114.8$.

(3-Nitrophenyl)(phenyl)tellane (3d). Yield: $0.137 \mathrm{~g}(84 \%)$; yellow oil; [55] ${ }^{1} \mathrm{H}-\mathrm{NMR}\left(400 \mathrm{MHz}, \mathrm{CDCl}_{3}\right.$, ppm) $\delta=8.42$ (s, $1 \mathrm{H}, \mathrm{H}-\mathrm{Ar}), 8.06$ (d, $J=8.2 \mathrm{~Hz}, 1 \mathrm{H}, \mathrm{H}-\mathrm{Ar}), 7.86-7.80$ (m, 3H, H-Ar), 7.41-7.25 (m, 4H, H-Ar); ${ }^{13} \mathrm{C}-\mathrm{NMR}\left(101 \mathrm{MHz}, \mathrm{CDCl}_{3}, \mathrm{ppm}\right) \delta=148.5,142.4,139.6,131.1,130.1,130.0,129.1,122.5$, 117.0, 113.2.

(2-Methoxyphenyl)(phenyl)tellane (3e). Yield: $0.140 \mathrm{~g}(90 \%)$; white solid; m.p.: $53-55^{\circ} \mathrm{C}$ (lit.: [48] $\left.53-54{ }^{\circ} \mathrm{C}\right) ;{ }^{1} \mathrm{H}-\mathrm{NMR}\left(200 \mathrm{MHz}, \mathrm{CDCl}_{3}, \mathrm{ppm}\right) \delta=7.89(\mathrm{dd}, J=8.1,1.4 \mathrm{~Hz}, 2 \mathrm{H}, \mathrm{H}-\mathrm{Ar}), 7.44-7.25$ (m, 3H, H-Ar), 7.17 (ddd, $J=8.2,7.3,1.6 \mathrm{~Hz}, 1 \mathrm{H}, \mathrm{H}-\mathrm{Ar}), 6.94$ (dd, $J=7.6,1.6 \mathrm{~Hz}, 1 \mathrm{H}, \mathrm{H}-\mathrm{Ar}), 6.80-6.69$ (m, 2H, H-Ar), 3.86 (s, 3H, $\left.-\mathrm{OCH}_{3}\right) ;{ }^{13} \mathrm{C}-\mathrm{NMR}\left(50 \mathrm{MHz}, \mathrm{CDCl}_{3}, \mathrm{ppm}\right) \delta=158.2,141.3,133.7,129.7$, $128.7,128.2,122.5,112.2,109.8,107.8,56.0$.

Naphthalen-2-yl(phenyl)tellane (3f). Yield: $0.146 \mathrm{~g}(88 \%)$; yellow oil; [55] ${ }^{1} \mathrm{H}-\mathrm{NMR}\left(200 \mathrm{MHz}, \mathrm{CDCl}_{3}\right.$, ppm) $\delta=8.19-8.14(\mathrm{~m}, 1 \mathrm{H}, \mathrm{H}-\mathrm{Ar}), 7.97(\mathrm{dd}, J=7.1,1.1 \mathrm{~Hz}, 1 \mathrm{H}, \mathrm{H}-\mathrm{Ar}), 7.82-7.74(\mathrm{~m}, 2 \mathrm{H}, \mathrm{H}-\mathrm{Ar}), 7.62-7.57$ (m, 2H, H-Ar), 7.51-7.44 (m, 2H, H-Ar), 7.28-7.08 (m, 4H, H-Ar); ${ }^{13} \mathrm{C}-\mathrm{NMR}$ (101 MHz, $\mathrm{CDCl}_{3}, \mathrm{ppm}$ ) $\delta=138.9,137.5,135.9,133.8,131.8,129.6,128.9,127.8,127.1,126.6,126.4,117.9,114.9$.

1-(4-((4-Methoxyphenyl)tellanyl)phenyl)ethan-1-one (3g). Yield: $0.152 \mathrm{~g}(86 \%)$; yellow oil; [57] ${ }^{1} \mathrm{H}-\mathrm{NMR}$ $\left(200 \mathrm{MHz}, \mathrm{CDCl}_{3}, \mathrm{ppm}\right) \delta=7.78(\mathrm{~d}, J=8.8 \mathrm{~Hz}, 2 \mathrm{H}, \mathrm{H}-\mathrm{Ar}), 7.68(\mathrm{~d}, J=8.5 \mathrm{~Hz}, 2 \mathrm{H}, \mathrm{H}-\mathrm{Ar})$, $7.48(\mathrm{~d}, J=8.5 \mathrm{~Hz}, 2 \mathrm{H}, \mathrm{H}-\mathrm{Ar}), 6.83(\mathrm{~d}, J=8.8 \mathrm{~Hz}, 2 \mathrm{H}, \mathrm{H}-\mathrm{Ar}), 3.82\left(\mathrm{~s}, 3 \mathrm{H},-\mathrm{OCH}_{3}\right), 2.52\left(\mathrm{~s}, 3 \mathrm{H},-\mathrm{COCH}_{3}\right)$; ${ }^{13} \mathrm{C}-\mathrm{NMR}\left(50 \mathrm{MHz}, \mathrm{CDCl}_{3}\right.$, ppm) $\delta=197.5,160.4,142.2,135.5,134.5,128.6,125.5,115.8,102.1,55.2,26.4$.

(3-Nitrophenyl)(p-tolyl)tellane (3h). Yield: $0.142 \mathrm{~g}(83 \%)$; yellow oil; [57] ${ }^{1} \mathrm{H}-\mathrm{NMR}\left(200 \mathrm{MHz}, \mathrm{CDCl}_{3}\right.$, ppm) $\delta=8.41-8.29$ (m, 1H, H-Ar), 8.06-8.01 (m, 1H, H-Ar), 7.82-7.72 (m, 3H, H-Ar), 7.30 (td, J = 8.3, $2.2 \mathrm{~Hz}, 1 \mathrm{H}, \mathrm{H}-\mathrm{Ar}), 7.12$ (d, J = 7.1 Hz, 2H, H-Ar), $2.38\left(\mathrm{~s}, 3 \mathrm{H},-\mathrm{CH}_{3}\right) ;{ }^{13} \mathrm{C}-\mathrm{NMR}\left(50 \mathrm{MHz}, \mathrm{CDCl}_{3}, \mathrm{ppm}\right)$ $\delta=148.5,141.8,140.1,139.5,131.0,130.4,129.8,122.2,117.6,109.1,21.4$.

1-(4-((4-Chlorophenyl)tellanyl)phenyl)ethanone (3i). Yield: $0.156 \mathrm{~g}(87 \%)$; yellow solid; m.p.: $67-68{ }^{\circ} \mathrm{C}$ (lit.: [57] 67-70 $\left.{ }^{\circ} \mathrm{C}\right) ;{ }^{1} \mathrm{H}-\mathrm{NMR}\left(200 \mathrm{MHz}, \mathrm{CDCl}_{3}, \mathrm{ppm}\right) \delta=7.76-7.72(\mathrm{~m}, 4 \mathrm{H}, \mathrm{H}-\mathrm{Ar}), 7.61(\mathrm{~d}, J=7.9 \mathrm{~Hz}$, 2H, H-Ar), 7.24 (d, J = 8.4 Hz, 2H, H-Ar), 2.56 (s, 3H, - $\left.\mathrm{COCH}_{3}\right) ;{ }^{13} \mathrm{C}-\mathrm{NMR}\left(50 \mathrm{MHz}, \mathrm{CDCl}_{3}, \mathrm{ppm}\right)$ $\delta=197.6,140.9,139.5,136.3,136.3,136.2,135.5,130.3,129.0,123.5,111.1,26.6$.

3-((4-Chlorophenyl)tellanyl)aniline (3j). Yield: $0.137 \mathrm{~g}$ (83\%); brown solid; m.p.: $60-61{ }^{\circ} \mathrm{C}$ (lit.: [57] $\left.58-60{ }^{\circ} \mathrm{C}\right) ;{ }^{1} \mathrm{H}-\mathrm{NMR}\left(200 \mathrm{MHz}, \mathrm{CDCl}_{3}, \mathrm{ppm}\right) \delta=7.58(\mathrm{~d}, J=8.2 \mathrm{~Hz}, 2 \mathrm{H}, \mathrm{H}-\mathrm{Ar}), 7.18-6.95$ (m, 5H, H-Ar), $6.59(\mathrm{dt}, J=7.5,1.9 \mathrm{~Hz}, 1 \mathrm{H}, \mathrm{H}-\mathrm{Ar}), 3.62\left(\mathrm{~s}, 2 \mathrm{H},-\mathrm{NH}_{2}\right) ;{ }^{13} \mathrm{C}-\mathrm{NMR}\left(50 \mathrm{MHz}, \mathrm{CDCl}_{3}, \mathrm{ppm}\right) \delta=147.4,139.2$, 134. 2, 130.4, 129.7, 128.2, 124.4, 115.0, 112.6.

1-(4-(Butyltellanyl)phenyl)ethan-1-one (3k). Yield: $0.127 \mathrm{~g}$ (87\%); yellow oil; [69] ${ }^{1} \mathrm{H}-\mathrm{NMR}(200 \mathrm{MHz}$, $\left.\mathrm{CDCl}_{3}, \mathrm{ppm}\right) \delta=7.67(\mathrm{~d}, J=8.8 \mathrm{~Hz}, 2 \mathrm{H}, \mathrm{H}-\mathrm{Ar}), 6.76(\mathrm{~d}, J=8.8 \mathrm{~Hz}, 2 \mathrm{H}, \mathrm{H}-\mathrm{Ar}), 3.79\left(\mathrm{~s}, 3 \mathrm{H},-\mathrm{OCH}_{3}\right)$, 2.86-2.78 (m, 2H, $\left.-\mathrm{CH}_{2}-\right), 1.73\left(\mathrm{p}, J=7.9,7.4 \mathrm{~Hz}, 2 \mathrm{H},-\mathrm{CH}_{2}-\right), 1.46-1.287\left(\mathrm{~m}, 2 \mathrm{H},-\mathrm{CH}_{2}-\right), 0.92-0.851$ $\left(\mathrm{m}, 3 \mathrm{H},-\mathrm{CH}_{3}\right) ;{ }^{13} \mathrm{C}-\mathrm{NMR}\left(101 \mathrm{MHz}, \mathrm{CDCl}_{3}, \mathrm{ppm}\right) \delta=159.7,141.0,115.2,100.7,55.2,34.0,25.1,13.5,8.9$.

(4-Methoxyphenyl)(phenyl)selane (6a). Yield: $0.114 \mathrm{~g}(87 \%)$; yellow oil; [54] ${ }^{1} \mathrm{H}-\mathrm{NMR}\left(200 \mathrm{MHz}, \mathrm{CDCl}_{3}\right.$, ppm) $\delta=7.51$ (d, $J=8.9 \mathrm{~Hz}, 2 \mathrm{H}, \mathrm{H}-\mathrm{Ar}), 7.35-7.18(\mathrm{~m}, 5 \mathrm{H}, \mathrm{H}-\mathrm{Ar}), 6.86(\mathrm{~d}, J=8.8 \mathrm{~Hz}, 2 \mathrm{H}, \mathrm{H}-\mathrm{Ar})$, $3.81\left(\mathrm{~s}, 3 \mathrm{H},-\mathrm{OCH}_{3}\right) ;{ }^{13} \mathrm{C}-\mathrm{NMR}\left(50 \mathrm{MHz}, \mathrm{CDCl}_{3}, \mathrm{ppm}\right) \delta=159.7,136.5,133.1,130.9,129.1,126.4,119.9$, 115.1, 55.3. 
(4-Chlorophenyl)(phenyl)selane (6b). Yield: $0.114 \mathrm{~g}(85 \%)$; transparent oil; [68 ${ }^{1} \mathrm{H}-\mathrm{NMR}\left(200 \mathrm{MHz}, \mathrm{CDCl}_{3}\right.$, ppm) $\delta=7.47-7.44$ (m, 2H, H-Ar), 7.35 (d, J = 8.3 Hz, 2H, H-Ar), 7.27-7.18 (m, 5H, H-Ar); ${ }^{13} \mathrm{C}-\mathrm{NMR}$ $\left(50 \mathrm{MHz}, \mathrm{CDCl}_{3}, \mathrm{ppm}\right) \delta=134.2,133.6,133.3,130.8,129.7,129.6,127.7$.

Phenyl(o-tolyl)selane (6c). Yield: $0.110 \mathrm{~g}(89 \%)$; transparent oil; [70] ${ }^{1} \mathrm{H}-\mathrm{NMR}\left(200 \mathrm{MHz}, \mathrm{CDCl}_{3}, \mathrm{ppm}\right)$ $\delta=7.42-7.36$ (m, 2H, H-Ar), 7.34-7.14 (m, 6H, H-Ar), 7.09-7.01 (m, 1H, H-Ar), 2.40 (s, 3H, - $\left.\mathrm{CH}_{3}\right)$; ${ }^{13} \mathrm{C}-\mathrm{NMR}\left(50 \mathrm{MHz}, \mathrm{CDCl}_{3}\right.$, ppm) $\delta=139.9,133.8,132.8,131.8,130.9,130.3,129.4,127.8,127.2,126.8,22.4$.

Naphthalen-2-yl(phenyl)selane (6d). Yield: $0.125 \mathrm{~g}(88 \%)$; yellow solid; m.p.: $68-70^{\circ} \mathrm{C}$ (lit.: [57] 69-71 ${ }^{\circ} \mathrm{C}$ ); ${ }^{1} \mathrm{H}-\mathrm{NMR}\left(200 \mathrm{MHz}, \mathrm{CDCl}_{3}\right.$, ppm) $\delta=8.35-8.30(\mathrm{~m}, 1 \mathrm{H}, \mathrm{H}-\mathrm{Ar}), 7.84-7.74(\mathrm{~m}, 3 \mathrm{H}, \mathrm{H}-\mathrm{Ar}), 7.51-7.15$ (m, $8 \mathrm{H}, \mathrm{H}-\mathrm{Ar}) ;{ }^{13} \mathrm{C}-\mathrm{NMR}\left(50 \mathrm{MHz}, \mathrm{CDCl}_{3}, \mathrm{ppm}\right) \delta=134.2,133.9,131.8,129.4,129.3,128.7,127.8,127.1$, $126.9,126.5,126.1$.

(4-Chlorophenyl)(4-methoxyphenyl)selane (6e). Yield: $0.132 \mathrm{~g}$ (89\%); yellow solid; m.p.: $56-57^{\circ} \mathrm{C}$ (lit.: [48] 58-59 $\left.{ }^{\circ} \mathrm{C}\right) ;{ }^{1} \mathrm{H}-\mathrm{NMR}\left(200 \mathrm{MHz}, \mathrm{CDCl}_{3}, \mathrm{ppm}\right) \delta=7.41$ (d, J = 8.8 Hz, 2H, H-Ar), 7.18-7.06 (m, 4H, H-Ar), $6.77(\mathrm{~d}, J=8.8 \mathrm{~Hz}, 2 \mathrm{H}, \mathrm{H}-\mathrm{Ar}), 3.72\left(\mathrm{~s}, 3 \mathrm{H},-\mathrm{OCH}_{3}\right) ;{ }^{13} \mathrm{C}-\mathrm{NMR}\left(50 \mathrm{MHz}, \mathrm{CDCl}_{3}, \mathrm{ppm}\right) \delta=160.0,136.6$, $132.5,132.1,131.6,129.2,119.5,115.2,55.3$.

(4-Chlorophenyl)(o-tolyl)selane (6f). Yield: $0.117 \mathrm{~g}(83 \%)$; transparent oil; [57] ${ }^{1} \mathrm{H}-\mathrm{NMR}\left(200 \mathrm{MHz}, \mathrm{CDCl}_{3}\right.$, ppm) $\delta=7.36-7.03(\mathrm{~m}, 8 \mathrm{H}, \mathrm{H}-\mathrm{Ar}), 2.37\left(\mathrm{~s}, 3 \mathrm{H},-\mathrm{CH}_{3}\right) ;{ }^{13} \mathrm{C}-\mathrm{NMR}\left(50 \mathrm{MHz}, \mathrm{CDCl}_{3}, \mathrm{ppm}\right) \delta=140.2,134.1$, 133.8, 133.4, 131.3, 130.5, 129.6, 129.4, 128.3, 127.0, 22.5 .

(4-Chlorophenyl)(o-tolyl)selane (6g). Yield: $0.130 \mathrm{~g}(89 \%)$; transparent oil; [57] ${ }^{1} \mathrm{H}-\mathrm{NMR}\left(200 \mathrm{MHz}, \mathrm{CDCl}_{3}\right.$, ppm) $\delta=7.55(\mathrm{~d}, J=8.8 \mathrm{~Hz}, 2 \mathrm{H}, \mathrm{H}-\mathrm{Ar}), 7.16-7.05(\mathrm{~m}, 1 \mathrm{H}, \mathrm{H}-\mathrm{Ar}), 6.88(\mathrm{~d}, J=8.8 \mathrm{~Hz}, 2 \mathrm{H}, \mathrm{H}-\mathrm{Ar}), 6.82-6.72$ $(\mathrm{m}, 3 \mathrm{H}, \mathrm{H}-\mathrm{Ar}), 3.88\left(\mathrm{~s}, 3 \mathrm{H},-\mathrm{OCH}_{3}\right), 3.81\left(\mathrm{~s}, 3 \mathrm{H},-\mathrm{OCH}_{3}\right) ;{ }^{13} \mathrm{C}-\mathrm{NMR}\left(50 \mathrm{MHz}, \mathrm{CDCl}_{3}, \mathrm{ppm}\right) \delta=160.3$, 156.0, 138.5, 129.1, 127.0, 123.6, 121.7, 117.4, 115.4, 110.2, 55.9, 55.4.

Butyl(4-methoxyphenyl)selane (4-chlorophenyl)(o-tolyl)selane (6h). Yield: $0.094 \mathrm{~g}$ (77\%); yellow oil; [48] ${ }^{1} \mathrm{H}-\mathrm{NMR}\left(200 \mathrm{MHz}, \mathrm{CDCl}_{3}, \mathrm{ppm}\right) \delta=7.46(\mathrm{~d}, J=8.9 \mathrm{~Hz}, 2 \mathrm{H}), 6.81(\mathrm{~d}, J=8.8 \mathrm{~Hz}, 2 \mathrm{H}), 3.79(\mathrm{~s}, 3 \mathrm{H}$, $\left.-\mathrm{OCH}_{3}\right), 2.82\left(\mathrm{t}, J=7.6 \mathrm{~Hz}, 2 \mathrm{H},-\mathrm{CH}_{2}-\right), 1.71-1.576\left(\mathrm{~m}, 2 \mathrm{H},-\mathrm{CH}_{2}-\right), 1.49-1.34\left(\mathrm{~m}, 2 \mathrm{H},-\mathrm{CH}_{2}-\right), 0.89$ $\left(\mathrm{t}, J=7.2 \mathrm{~Hz}, 3 \mathrm{H},-\mathrm{CH}_{3}\right) ;{ }^{13} \mathrm{C}-\mathrm{NMR}\left(50 \mathrm{MHz}, \mathrm{CDCl}_{3}, \mathrm{ppm}\right) \delta=159.2,135.6,120.4,114.8,55.4,32.5,29.0$, 23.0, 13.7.

(4-Methoxyphenyl)(phenyl)sulfane (7a). Yield: $0.077 \mathrm{~g}(71 \%)$; transparent oil; [68] ${ }^{1} \mathrm{H}-\mathrm{NMR}(200 \mathrm{MHz}$, $\left.\mathrm{CDCl}_{3}, \mathrm{ppm}\right) \delta=7.42$ (d, J= 8.9 Hz, 2H, H-Ar), 7.28-7.15 (m, 5H, H-Ar), 6.90 (d, J = 8.9 Hz, 2H, H-Ar), $3.82\left(\mathrm{~s}, 3 \mathrm{H},-\mathrm{OCH}_{3}\right) ;{ }^{13} \mathrm{C}-\mathrm{NMR}\left(50 \mathrm{MHz}, \mathrm{CDCl}_{3}, \mathrm{ppm}\right) \delta=160.0,138.7,135.5,129.0,128.3,125.9,124.5$, $115.1,55.5$.

(3-Chlorophenyl)(4-methoxyphenyl)sulfane (7b). Yield: $0.085 \mathrm{~g}(68 \%)$; white solid; m.p.: 59-61 ${ }^{\circ} \mathrm{C}$ (lit.: [57] 59-61 $\left.{ }^{\circ} \mathrm{C}\right) ;{ }^{1} \mathrm{H}-\mathrm{NMR}\left(400 \mathrm{MHz}, \mathrm{CDCl}_{3}, \mathrm{ppm}\right) \delta=7.42(\mathrm{~d}, J=8.9 \mathrm{~Hz}, 2 \mathrm{H}, \mathrm{H}-\mathrm{Ar}), 7.13-7.03(\mathrm{~m}, 3 \mathrm{H}$, $\mathrm{H}-\mathrm{Ar}), 6.98$ (dt, J = 7.7, $1.3 \mathrm{~Hz}, 1 \mathrm{H}, \mathrm{H}-\mathrm{Ar}), 6.91$ (d, $J=8.9 \mathrm{~Hz}, 2 \mathrm{H}, \mathrm{H}-\mathrm{Ar}), 3.81$ (s, 3H, $\left.-\mathrm{OCH}_{3}\right) ;{ }^{13} \mathrm{C}-\mathrm{NMR}$ $\left(50 \mathrm{MHz}, \mathrm{CDCl}_{3}, \mathrm{ppm}\right) \delta=160.4,141.4,136.2,134.9,129.9,127.2,125.7,125.6,122.8,115.4,55.5$.

\section{Conclusions}

In summary, we have developed a straightforward method for the preparation of unsymmetrical diorganyl chalcogenides (S, Se and Te), under solvent- and ligand-free conditions. Remarkably, copper iodide was found to be a highly efficient catalyst for this cross-coupling reaction, affording the desired products in very high yields in the absence of solvent under microwave irradiation. Of particular importance, this new metal-catalyzed process showed good potential applicability within a very broad scope, without the need for the use of ligands or solvents. It appears that the chemistry described herein characterizes a useful alternative approach to the synthesis of organochalcogen compounds through a copper-catalyzed processes.

Supplementary Materials: Copies of the ${ }^{1} \mathrm{H}$ - and ${ }^{13} \mathrm{C}-\mathrm{NMR}$ spectra are available online. 
Acknowledgments: We gratefully acknowledge the CNPq, CAPES, INCT-Catálise, FAPESP-CERSusChem (grant 2014/50249-8), GSK-CERSusChem (grant 2014/50249-8), HEC (SRGP-161), FAPESC-Pronex, and FAPERGS-Pronex, for financial support. CAPES is also acknowledged for the fellowship for J.R. under CAPES-PNPD scheme. The funding bodies had no role in the study design, data collection and analysis, decision to publish, or preparation of the manuscript.

Author Contributions: A.L.B., S.S., J.R., G.V.B. conceived and designed the experiments; S.S., J.R., G.V.B. and T.E.A.F. performed the experiments; S.S., J.R., G.V.B., M.G., T.E.A.F. and F.Z.G. performed the spectral analysis, characterizations and contributed reagents/materials; A.L.B., S.S. and J.R. wrote the paper. All authors have approved the final version of the manuscript.

Conflicts of Interest: The authors declare no conflict of interest.

\section{References}

1. Liu, C.; Zhang, H.; Shi, W.; Lei, A. Bond formations between two nucleophiles: Transition metal catalyzed oxidative cross-coupling reactions. Chem. Rev. 2011, 111, 1780-1824. [CrossRef] [PubMed]

2. Yang, L.; Huang, H. Transition-metal-catalyzed direct addition of unactivated $\mathrm{C}-\mathrm{H}$ bonds to polar unsaturated bonds. Chem. Rev. 2015, 115, 3468-3517. [CrossRef] [PubMed]

3. Li, Y.; Nie, C.; Wang, H.; Li, X.; Verpoort, F.; Duan, C. A highly efficient method for the copper-catalyzed selective synthesis of diaryl chalcogenides from easily available chalcogen sources. Eur. J. Org. Chem. 2011, 2011, 7331-7338. [CrossRef]

4. Sun, J.; Deng, L. Cobalt complex-catalyzed hydrosilylation of alkenes and alkynes. ACS Catal. 2017, 7, 631-651. [CrossRef]

5. Chen, Z.; Huang, J.; Wang, Z. Transition-metal-catalyzed hydrosulfoximination and oxidation reaction for the synthesis of sulfoximine derivatives. J. Org. Chem. 2016, 81, 9308-9314. [CrossRef] [PubMed]

6. Liu, C.; Szostak, M. Twisted amides: From obscurity to broadly useful transtition-metal-catalyzed reaction by N-C amide bond Activation. Chem. Eur. J. 2017, 23, 7157-7173. [CrossRef] [PubMed]

7. Meng, G.; Shi, S.; Szostak, M. Cross-coupling of amides by N-C bond activation. Synlett 2016, 27, $2530-2540$.

8. Godoi, M.; Paixão, M.W.; Braga, A.L. Chiral organoselenium-transition-metal catalysts in asymmetric transformations. Dalton Trans. 2011, 40, 11347-11355. [CrossRef] [PubMed]

9. Cresswell, A.J.; Eey, S.T.-C.; Denmark, S.E. Catalytic, stereospecific syn-dichlorination of alkenes. Nat. Chem. 2015, 7, 146-152. [CrossRef] [PubMed]

10. Prochnow, T.; Back, D.F.; Geni, Z. Iron(III) chloride and diorganyl diselenide-promoted nucleophilic closures of 1-benzyl-2-alkynylbenzenes in the preparation of 9-(organoselanyl)-5H-benzo[7]annulenes. Adv. Synth. Catal. 2016, 358, 1119-1129. [CrossRef]

11. Rafique, J.; Canto, R.F.S.; Saba, S.; Barbosa, F.A.R.; Braga, A.L. Recent advances in the synthesis of biologically relevant selenium-containing 5-membered heterocycles. Curr. Org. Chem. 2016, 20, 166-188. [CrossRef]

12. Manna, D.; Roy, G.; Mugesh, G. Antithyroid drugs and their analogues: Synthesis, structure, and mechanism of action. Acc. Chem. Res. 2013, 46, 2706-2715. [CrossRef] [PubMed]

13. Barbosa, F.A.R.; Canto, R.F.S.; Saba, S.; Rafique, J.; Braga, A.L. Synthesis and evaluation of dihydropyrimidinone-derived selenoesters as multi-targeted directed compounds against Alzheimer's disease. Bioorg. Med. Chem. 2016, 24, 5762-5770. [CrossRef] [PubMed]

14. Kumar, S.; Yan, J.; Poon, J.-F.; Singh, V.P.; Lu, X.; Ott, M.K.; Engman, L.; Kumar, S. Multifunctional antioxidants: Regenerable radical-trapping and hydroperoxide-decomposing ebselenols. Angew. Chem. Int. Ed. 2016, 55, 3729-3733. [CrossRef] [PubMed]

15. Rafique, J.; Saba, S.; Canto, R.F.S.; Frizon, T.E.A.; Hassan, W.; Waczuk, E.P.; Jan, M.; Back, D.F.; Rocha, J.B.T.D.; Braga, A.L. Synthesis and biological evaluation of 2-picolylamide-based diselenides with non-bonded interactions. Molecules 2015, 20, 10095-10109. [CrossRef] [PubMed]

16. Freudendahl, D.M.; Santoro, S.; Shahzad, S.A.; Santi, C.; Wirth, T. Green chemistry with selenium reagents: Development of efficient catalytic reactions. Angew. Chem. Int. Ed. 2009, 48, 8409-8411. [CrossRef] [PubMed]

17. Braga, A.L.; Rafique, J. Synthesis of biologically relevant small molecules containing selenium. Part A. Antioxidant compounds. In The Chemistry of Organic Selenium and Tellurium Compounds; Rappoport, Z., Ed.; Wiley \& Sons, Ltd.: West Sussex, UK, 2014; Volume 4, Chapter 13, pp. 989-1052. 
18. Braga, A.L.; Rafique, J. Synthesis of biologically relevant small molecules containing selenium. Part B. Anti-infective and anticancer compounds. In The Chemistry of Organic Selenium and Tellurium Compounds; Rappoport, Z., Ed.; Wiley \& Sons, Ltd.: West Sussex, UK, 2014; Volume 4, Chapter 14, pp. 1053-1118.

19. Braga, A.L.; Rafique, J. Synthesis of biologically relevant small molecules containing selenium. Part C. Miscellaneous biological activities. In The Chemistry of Organic Selenium and Tellurium Compounds; Rappoport, Z., Ed.; Wiley \& Sons, Ltd.: West Sussex, UK, 2014; Volume 4, Chapter 15, pp. 1119-1174.

20. Frizon, T.E.; Rafique, J.; Saba, S.; Bechtold, I.H.; Gallardo, H.; Braga, A.L. Synthesis of functionalized organoselenium materials: Selenides and diselenides containing cholesterol. Eur. J. Org. Chem. 2015, 2015, 3470-3476. [CrossRef]

21. Brutchey, R.L. Diorganyl dichalcogenides as useful synthons for colloidal semiconductor nanocrystals. Acc. Chem. Res. 2015, 48, 2918-2926. [CrossRef] [PubMed]

22. Ho, P.C.; Rafique, J.; Lee, J.; Lee, L.M.; Jenkins, H.A.; Britten, J.F.; Braga, A.L.; Vargas-Baca, I. Synthesis and structural characterisation of the aggregates of benzo-1,2-chalcogenazole 2-oxides. Dalton Trans. 2017, 46, 6570-6579. [CrossRef] [PubMed]

23. Gu, J.; Zhao, Z.-Q.; Ding, Y.; Chen, H.-L.; Zhang, Y.-W.; Yan, C.-H. Liquid-phase syntheses and material properties of two-dimensional nanocrystals of rare earth-selenium compound containing planar se layers: $\mathrm{RESe}_{2}$ nanosheets and $\mathrm{RE}_{4} \mathrm{O}_{4} \mathrm{Se}_{3}$ nanoplates. J. Am. Chem. Soc. 2013, 135, 8363-8371. [CrossRef] [PubMed]

24. Godoi, B.; Schumacher, R.F.; Zeni, G. Synthesis of heterocycles via electrophilic cyclization of alkynes containing heteroatom. Chem. Rev. 2011, 111, 2937-2980. [CrossRef] [PubMed]

25. Bhabak, K.P.; Mughesh, G. Functional mimics of glutathione peroxidase: Bioinspired synthetic antioxidants. Acc. Chem. Res. 2010, 43, 1408-1419. [CrossRef] [PubMed]

26. Zeni, G.; Braga, A.L.; Stefani, H.A. Palladium-catalyzed coupling of $\mathrm{sp}^{2}$-hybridized tellurides. Acc. Chem. Res. 2003, 36, 731-738. [CrossRef] [PubMed]

27. Wirth, T. Organoselenium Chemistry: Synthesis and Reactions; Wiley-VCH: Weinheim, Germany, 2011.

28. Rappoport, Z.; Liebman, J.F.; Marek, I.; Patai, S. The Chemistry of Organic Selenium and Tellurium Compounds; Wiley \& Sons, Ltd.: West Sussex, UK, 2014; Volume 4.

29. Rappoport, Z.; Liebman, J.F.; Marek, I.; Patai, S. The Chemistry of Organic Selenium and Tellurium Compounds; Wiley \& Sons, Ltd.: West Sussex, UK, 2012; Volume 3.

30. Zeni, G.; Lüdtke, D.S.; Panatieri, R.B.; Braga, A.L. Vinylic tellurides: From preparation to their applicability in organic synthesis. Chem. Rev. 2006, 106, 1032-1076. [CrossRef] [PubMed]

31. Mugesh, G.; Singh, H.B. Heteroatom-directed aromatic lithiation: A versatile route to the synthesis of organochalcogen (Se, Te) compounds. Acc. Chem. Res. 2002, 35, 226-236. [CrossRef] [PubMed]

32. Kumar, S.; Singh, H.B.; Wolmershäuser, G. Protection against peroxynitrite-mediated nitration reaction by intramolecularly coordinated diorganoselenides. Organometallics 2006, 25, 382-393. [CrossRef]

33. Malysher, D.A.; Scott, N.M.; Marion, N.; Stevens, E.D.; Ananikov, V.P.; Beletskaya, I.P.; Nolan, S.P. Homogeneous nickel catalysts for the selective transfer of a single arylthio group in the catalytic hydrothiolation of alkynes. Organometallics 2006, 25, 4462-4470. [CrossRef]

34. Cohen, R.J.; Fox, D.L.; Salvatore, R.N. A novel and highly efficient synthetic route to unsymmetrical organoselenides using cesium bases. J. Org. Chem. 2004, 69, 4265-4268. [CrossRef] [PubMed]

35. Bates, C.G.; Gujadhur, R.K.; Venkataraman, D. A general method for the formation of aryl-sulfur bonds using copper (I) catalysts. Org. Lett. 2002, 4, 2803-2806. [CrossRef] [PubMed]

36. Sperotto, E.; van Klink, G.P.M.; de Vries, J.G.; van Koten, G. Ligand-free copper-catalyzed c-s coupling of aryl iodides and thiols. J. Org. Chem. 2008, 73, 5625-5628. [CrossRef] [PubMed]

37. Tiecco, M.; Testaferri, L.; Bagnoli, L.; Mariani, F.; Temperini, A.; Tomassini, C.; Santi, C. Electrophilic 2-thienylselenenylation of thiophene. Preparation of oligo(seleno-2,5-thienylenes). Tetrahedron 2000, 56, 3255-3260. [CrossRef]

38. Jin, W.; Zheng, P.; Wong, W.-T.; Law, G.-L. Efficient palladium-catalyzed direct C-H phenylselenylation of (hetero)arenes in water. Asian J. Org. Chem. 2015, 4, 875-878. [CrossRef]

39. Cheng, J.-H.; Yi, C.-L.; Liu, T.-J.; Lee, C.-F. Highly regioselective synthesis of aryl chalcogenides through C-H functionalization of arenes. Chem. Commun. 2012, 48, 8440-8442. [CrossRef] [PubMed]

40. Chu, L.; Yue, X.; Qing, F.-L. Cu (II)-mediated methylthiolation of aryl C-H bonds with DMSO. Org. Lett. 2010, 12, 1644-1647. [CrossRef] [PubMed] 
41. Zhao, X.; Dimitrijević, E.; Dong, V.M. Palladium-catalyzed C-H Bond functionalization with arylsulfonyl chlorides. J. Am. Chem. Soc. 2009, 131, 3466-3467. [CrossRef] [PubMed]

42. Candeias, N.R.; Montlbano, F.; Cal, P.M.S.D.; Gois, P.M.P. Boronic acids and esters in the petasis-borono Mannich multicomponent reaction. Chem. Rev. 2010, 110, 6169-6193. [CrossRef] [PubMed]

43. Zhu, C.; Falck, J.R. Transition-Metal-Free ipso-Functionalization of Arylboronic Acids and Derivatives. Adv. Synth. Catal. 2014, 356, 2395-2410. [CrossRef] [PubMed]

44. Hall, D.G. Boronic Acids: Preparation and Applications in Organic Synthesis Medicine and Materials, 2nd ed.; Wiley-VCH: Weinheim, Germany, 2011; Volume 2.

45. Taniguchi, N. Aryl- or alkylation of diaryl disulfides using organoboronic acids and a copper catalyst. Synlett 2006, 14, 1351-1354. [CrossRef]

46. Wang, L.; Wang, M.; Huang, F. A simple copper salt-catalyzed synthesis of unsymmetrical diaryl selenides and tellurides from arylboronic acids with diphenyl diselenide and ditelluride. Synlett 2005, 13, 2007-2010. [CrossRef]

47. Ren, K.; Wang, M.; Wang, L. Lewis acid InBr3-catalyzed arylation of diorgano diselenides and ditellurides with arylboronic acids. Org. Biomol. Chem. 2009, 7, 4858-4861. [CrossRef] [PubMed]

48. Wang, M.; Ren, K.; Wang, L. Iron-catalyzed ligand-free carbon-selenium (or tellurium) coupling of arylboronic acids with diselenides and ditellurides. Adv. Synth. Catal. 2009, 351, 1586-1594. [CrossRef]

49. Yu, J.-T.; Guo, H.; Yi, Y.; Fei, H.; Jiang, Y. The Chan-Lam reaction of chalcogen elements leading to aryl chalcogenides. Adv. Synth. Catal. 2014, 356, 749-752. [CrossRef]

50. Zheng, B.; Gong, Y.; Xu, H.-H. Copper-catalyzed C-Se coupling of diphenyl diselenide with arylboronic acids at room temperature. Tetrahedron 2013, 69, 5342-5347. [CrossRef]

51. Reddy, K.H.; Satish, G.; Rames, K.; Karnakar, K.; Nageswar, Y.V.D. Magnetically separable $\mathrm{CuFe}_{2} \mathrm{O}_{4}$ nanoparticle catalyzed C-Se cross coupling in reusable PEG medium. Chem. Lett. 2012, 41, 585-587. [CrossRef]

52. Mohan, B.; Yoon, C.; Jang, S. Copper nanoparticles catalyzed Se(Te)-Se(Te) bond activation: A straightforward route towards unsymmetrical organochalcogenides from boronic acids. Chemcatchem 2015, 7, 405-412. [CrossRef]

53. Guo, Y.; Quan, Z.-J.; Da, Y.-X.; Zhang, Z.; Wang, X.-C. (2-Chlorobenzoyloxy)copper(I) catalyzed C-S cross-coupling of di(hetero)aryl disulfides with aryl boronic acis under base-free conditions. RSC Adv. 2015, 4, 45479-45483. [CrossRef]

54. Alves, D.; Santos, C.G.; Paixão, M.W.; Soares, L.C.; de Souza, D.; Rodrigues, O.E.D.; Braga, A.L. CuO nanoparticles: An efficient and recyclable catalyst for cross-coupling reactions of organic diselenides with aryl boronic acids. Tetrahedron Lett. 2009, 50, 6635-6638. [CrossRef]

55. Kumar, A.; Kumar, S. A convenient and efficient copper-catalyzed synthesis of unsymmetrical and symmetrical diaryl chalcogenides from arylboronic acids in ethanol. Tetrahedron 2014, 70, 1763-1772. [CrossRef]

56. Goldani, B.; Ricordi, V.G.; Seus, N.; Lenardao, E.J.; Schumacher, R.F.; Alves, D. Silver-catalyzed synthesis of diaryl selenides by reaction of diaryl diselenides with aryl boronic acids. J. Org. Chem. 2016, 81, 11472-11476. [CrossRef] [PubMed]

57. Saba, S.; Rafique, J.; Braga, A.L. Synthesis of unsymmetrical diorganyl chalcogenides under greener conditions: Use of an iodine/DMSO system, solvent- and metal-free approach. Adv. Synth. Catal. 2015, 357, 1446-1452. [CrossRef]

58. Mohan, B.; Hwang, S.; Jang, S.; Park, K.H. Ultrasound-assisted transition-metal-free synthesis of diaryl tellurides from aryl boronic acids: A possible free-radical mechanism. Syntlett 2014, 25, 2078-2083. [CrossRef]

59. Rafique, J.; Saba, S.; Rosário, A.R.; Braga, A.L. Regioselective, solvent- and metal-free chalcogenation of imidazo[1,2-a]pyridines by employing $\mathrm{I}_{2}$ /DMSO as the catalytic oxidation system. Chem. Eur. J. 2016, 22, 11854-11862. [CrossRef] [PubMed]

60. Saba, S.; Rafique, J.; Braga, A.L. DMSO/iodine-catalyzed oxidative C-Se/C-S bond formation: A regioselective synthesis of unsymmetrical chalcogenides with nitrogen- or oxygen-containing arenes. Catal. Sci. Technol. 2016, 6, 3087-3098. [CrossRef]

61. Rafique, J.; Saba, S.; Rosário, A.R.; Zeni, G.; Braga, A.L. $\mathrm{K}_{2} \mathrm{CO}_{3}$-mediated, direct C-H bond selenation and thiolation of 1,3,4-oxadiazoles in the absence of metal catalyst: An eco-friendly approach. RSC Adv. 2014, 4, 51648-51652. [CrossRef] 
62. Silva, L.T.; Azeredo, J.B.; Saba, S.; Rafique, J.; Bortoluzzi, A.J.; Braga, A.L. Solvent- and metal-free chalcogenation of bicyclic arenes using $\mathrm{I}_{2} / \mathrm{DMSO}$ as non-metallic catalytic system. Eur. J. Org. Chem. 2017. [CrossRef]

63. Rocha, M.S.T.; Rafique, J.; Saba, S.; Azeredo, J.B.; Back, D.; Godoi, M.; Braga, A.L. Regioselective hydrothiolation of terminal acetylene catalyzed by magnetite $\left(\mathrm{Fe}_{3} \mathrm{O}_{4}\right)$ nanoparticles. Synth. Commun. 2017, 47, 291-298. [CrossRef]

64. Rafique, J.; Saba, S.; Schneider, A.R.; Franco, M.S.; Silva, S.M.; Braga, A.L. Metal- and solvent-free approach to access 3-Se/S-chromones from the cyclization of enaminones in the presence of dichalcogenides catalyzed by $\mathrm{KIO}_{3}$. ACS Omega 2017, 2, 2280-2290. [CrossRef]

65. Ananikov, V.P.; Gayduk, K.A.; Belestskaya, I.P.; Khrustalve, V.N.; Antipin, M.Y. Remarkable ligand effect in Ni- and Pd-catalyzed bisthiolation and bisselenation of terminal alkynes: Solving the problem of stereoselective dialkyldichalcogenide addition to the $\mathrm{C} \equiv \mathrm{C}$ bond. Chem. Eur. J. 2008, 14, 2420-2434. [CrossRef] [PubMed]

66. Taniguchi, N. Diarylation of chalcogen elements using arylboronic acids via copper- or palladium-catalyzed oxidative coupling. Tetrahedron 2016, 72, 5818-5823. [CrossRef]

67. Zhao, H.; Jiang, Y.; Chen, Q.; Cai, M. A highly efficient and reusable MCM-41-immobilized bipyridine copper (I) catalyst for the C-Se coupling of organoboronic acids with diaryl diselenides. New J. Chem. 2015, 39, 2106-2115. [CrossRef]

68. Taniguchi, N. Convenient synthesis of unsymmetrical organochalcogenides using organoboronic acids with dichalcogenides via cleavage of the S-S, Se-Se, or Te-Te bond by a copper catalyst. J. Org. Chem. 2007, 72, 1241-1245. [CrossRef] [PubMed]

69. Cella, R.; Cunha, R.L.O.R.; Reis, A.E.S.; Pimenta, D.C.; Klitzke, C.F.; Stefani, H.A. Suzuki-Miyaura cross-coupling reactions of aryl tellurides with potassium aryltrifluoroborate salts. J. Org. Chem. 2006, 71, 244-250. [CrossRef] [PubMed]

70. Ricordi, V.G.; Freitas, C.S.; Perin, G.; Lenardão, E.J.; Jacob, R.G.; Savegnago, L.; Alves, D. Glycerol as a recyclable solvent for copper-catalyzed cross-coupling reactions of diaryl diselenides with aryl boronic acids. Green Chem. 2012, 14, 1030-1034. [CrossRef]

Sample Availability: Samples of the compounds $3 \mathbf{a}-\mathbf{k}, \mathbf{6} \mathbf{\mathbf { a }}-\mathbf{h}$ and $\mathbf{7 a}-\mathbf{b}$ are available from the authors.

(C) 2017 by the authors. Licensee MDPI, Basel, Switzerland. This article is an open access article distributed under the terms and conditions of the Creative Commons Attribution (CC BY) license (http:/ / creativecommons.org/licenses/by/4.0/). 